\title{
Anti-NMDA Receptor Encephalitis
}

\author{
Ajay Goenka ${ }^{1,2} \cdot$ Vivek Jain $^{3}$
}

Received: 13 December 2015 / Accepted: 5 May 2016/Published online: 21 May 2016

(C) Dr. K C Chaudhuri Foundation 2016

To the Editor: Although pediatric anti-N-methyl-D-aspartate receptor (NMDA-R) encephalitis is considered a rare disease, recent reports suggest that it is likely an under recognized cause of encephalitis [1]. Herein we briefly describe five cases of pediatric anti-NMDA-R encephalitis from a single medical center in India to emphasize and highlight the extended clinical spectrum of this disorder.

Out of five cases, four were girls. The median age was $2.5 \mathrm{y}$ (range 5 mo to $11 \mathrm{y}$ ). The presenting clinical symptoms included irritability (case 1), sleep disturbance and autonomic instability (case 2), altered mental status (case 3), non-convulsive focal status epilepticus (case 4) and isolated expressive aphasia (case 5). Four patients were observed to have noticeable choreiform movements. All patients had electrographic slowing or seizure patterns on EEG but no delta brush patterns were evident. All brain MRI and CSF studies were unremarkable. One patient (case 3) had positive HSV-1 CSF titers from recently treated herpes simplex virus (HSV) encephalitis. Diagnosis of antiNMDA-R encephalitis was confirmed by CSF and serum antiNMDA-R antibodies against the NR1 subunit. Screening for primary malignancy was negative in all cases. Four patients

The patient was admitted and treated at S.D.M.H. Jaipur under Dr. Vivek Jain.

Ajay Goenka

agoenka@montefiore.org

1 Montefiore Medical Centre, Albert Einstein College of Medicine, Yeshiva University, Bronx, NY 10467, USA

2 Saul Korey Department of Neurology, Children Hospital at Montefiore, Fourth Floor, 3415 Bainbridge Avenue, Bronx, NY 10467, USA

3 Department of Pediatric Neurology, Santokba Durlabhji Memorial Hospital, Jaipur, Rajasthan, India received IVIG and methylprednisolone, three received rituximab and one received cyclophosphamide. One patient recovered spontaneously without treatment. Complete recovery was seen in two patients while two others had mild residual neurological deficits. One patient died from autonomic instability. One patient had a disease relapse after 6 mo and was treated with rituximab.

There are no standard guidelines for treatment of NMDA-R encephalitis in the adult or pediatric populations. Expert consensus recommendations include intravenous steroids and/or IVIG as first line therapy followed by rituximab alone or in combination with cyclophosphamide when the treatment response to rituximab alone is unsatisfactory [2]. Longitudinal malignancy screening is always recommended.

This case series underscores the highly heterogeneous clinical phenotype of NMDA-R encephalitis in the pediatric population. Diagnostic vigilance should be maintained in all cases of suspected NMDA-R encephalitis as early intervention may prove vital in improving outcomes of this devastating neurological condition.

\section{Compliance with Ethical Standards}

Conflict of Interest None.

Source of Funding None.

\section{References}

1. Florance NR, Davis RL, Lam C, et al. Anti-N-methyl-D-aspartate receptor (NMDAR) encephalitis in children and adolescents. Ann Neurol. 2009;66:11-8.

2. Dalmau J, Lancaster E, Martinez-Hernandez E, Rosenfeld MR, Balice-Gordon R. Clinical experience and laboratory investigations in patients with anti-NMDAR encephalitis. Lancet Neurol. 2011;10: 63-74. 